\section{Composição corporal e maturação somática de meninas atletas e não-atletas de natação da cidade do Recife, Brasil}

\author{
Body composition and somatic maturation \\ of athletes and non-athlete of swimmers \\ girls from Recife city, Brazil
}

Marcos André Moura dos Santos1

Carol Góis Leandro2

Fernando José de Sá Guimarães3
1 Faculdade de Ciências do Desporto e de Educação Física. Universidade do Porto. Rua D. Manuel II 4000. Porto, Portugal. E-mail: mmoura23@gmail.com

2,3 Escola Superior de Educação Física. Universidade de Pernambuco. Recife, Brasil

\begin{abstract}
Objectives: to describe body composition alteration patterns (body weight, height, body mass index - BMI), percentage of body fat and skinfold thickness, and to compare menarche's age of athletes and nonathletes.

Methods: we evaluated 165 girls (aged 9 to 17 years old) non-athletes and athletes of swimmers grouped according to maturational groups (prepuberty, puberty e post-puberty). The anthropometric variables followed standardized rules. The percentage of body fat was estimated by an equation validated for the studied population. The age of menarche was established through the record method.

Results: there was no difference between groups in the pre-puberty stage. In the puberty stage, the athletes showed lower triciptal skinfold mean values $($ athletes $=15.5 \pm 15.3 ;$ non-athletes $=20.1 \pm 7.0, p$ $<0.05$ ), and subscapular (athletes $=14.7 \pm 5.1 \mathrm{e}$ non-athletes $=20.6 \pm 10.3, p<0.05)$, percentage of body fat (athletes $=25.2 \pm 5.9$ e non- athletes $=28.4$ $6.0, p<0.05$ ), and the fat mass (athletes $=8.7 \pm 3.5$ and non-athletes $=9.8 \pm 3.7, p<0.05)$. These results remained in the post-puberty stage. Menarche occurred later in the athlete group (athletes $=12,0 \pm$ 1,04 e non-athletes $=11,2 \pm 1,37, p<0.05$ ).

Conclusions: in athletes, some changes in body composition seem to take place, particularly the amount of body fat which could be associated with late menarche.
\end{abstract}

Key words Body fat, Growth, Training, Menarche

\section{Resumo}

Objetivos: descrever os valores médios de variáveis antropométricas (peso, estatura e indice de massa corporal - IMC), percentual de gordura corporal $e$ valores médios das dobras cutâneas e comparar a idade da menarca entre os grupos de meninas atletas e nãoatletas.

Métodos: foram avaliadas 165 meninas (entre os $9 e$ 17 anos) atletas e não-atletas de natação, divididas em grupos maturacionais (pré-púberes, púberes e póspúberes). A avaliação das variáveis antropométricas seguiu normas padronizadas. O percentual de gordura foi estimado a partir de equação validada para a população estudada. A idade de menarca foi determinada através do método recordatório.

Resultados: não houve diferença entre os grupos na fase pré-púbere. Na fase púbere, as atletas apresentaram um menor valor médio da dobra cutânea tricipital (atletas $=15,5 \pm 15,3$; não-atletas $=20.15 \pm 7.0, p<$ 0.05 ) e subescapular (atletas $=14,7$ 5,1 e não-atletas $=$ $20,6 \pm 10,3 ; p<0,05)$, do percentual de gordura corporal (atletas $=25,2 \pm 5,9$ e não-atletas $=28,4 \pm$ 6,0; $p<0.05$ ) e da massa gorda (atletas $=8,7 \pm 3,5$ e não-atletas $=9,8 \pm 3,7 ; p<0,05)$. Esses resultados permaneceram na fase pós-púbere. A idade da menarca das atletas foi mais tardia (atletas $=12,0 \pm 1,04$ e nãoatletas $=11,2 \pm 1,37 ; p<0,05)$.

Conclusões: em atletas, parece haver alterações na composição corporal, particularmente a quantidade de gordura corporal, podendo ser associadas ao aparecimento tardio da menarca.

Palavras-chave Gordura corporal, Crescimento, Treinamento, Menarca 


\section{Introdução}

A avaliação da composição corporal permite a quantificação dos componentes estruturais do corpo. ${ }^{1}$ Considerar aspectos maturacionais na composição corporal é imprescindível na interpretação das tendências fisiológicas peculiares à criança em crescimento. 2

O crescimento somático e a maturação física são processos dinâmicos associados a um amplo espectro de alterações somáticas e celulares. ${ }^{2}$ Embora as mudanças morfológicas e fisiológicas, típicas do processo maturacional, decorram de forma contínua, existe uma proposta de divisão em séries de estágios pubertais de desenvolvimento baseados nas características sexuais secundárias. ${ }^{3} \mathrm{O}$ tempo médio em que o indivíduo progride nesses estágios pubertais é cerca de quatro anos. ${ }^{2}$ Este processo ocorre primeiro nas meninas, normalmente com o desenvolvimento mamário (telarca), começando aos 10,5 ou 11 anos de idade e culmina com o surgimento da menarca. 3 Para os rapazes, o aumento da genitália começa em média entre os 11 ou 12,5 anos de idade.2,3

O crescimento somático e a maturação física podem ser influenciados por inúmeros fatores que atuam, independentemente ou em conjunto, para alterar o potencial genético individual. ${ }^{1} \mathrm{O}$ estado nutricional, incluindo a injesta de nutrientes específicos, parece ser o principal determinante do crescimento. ${ }^{4}$ Por exemplo, a subnutriçao está associada a um aparecimento tardio da menarca bem como amenorréia secundária. 5 Por outro lado, a obesidade está associada com uma maturação sexual antecipada. 5

Mais recentemente, o efeito do exercício físico sobre o crescimento, especialmente em jovens atletas, tem se tornado um tópico de interesse.1,6,7 Particularmente as meninas estão cada vez mais envolvidas em atividades físicas tanto no âmbito do lazer como da competição. ${ }^{7} \mathrm{Na}$ competição, a prática de exercício físico é realizada regularmente através do treinamento e segue uma estreita programação quanto à intensidade, duração, freqüência e tipo do esforço. ${ }^{8}$ Toda essa programação de exercício é realizada com o objetivo de rendimento esportivo, e os indivíduos engajados nesses pro-gramas são chamados de atletas. ${ }^{7,8}$

As meninas engajadas em esportes como a ginástica olímpica, balé, natação e saltos ornamentais iniciam os programas de treinamento ainda na idade infantil.9,10 Alguns aspectos da composição corporal parecem ser atingidos repercutindo diretamente no processo maturacional.2,9 A exemplo disso, Thomis et al. ${ }^{7}$ realizaram um estudo longitudinal com atletas de ginástica belgas dos 8 aos 15 anos. Esses autores observaram que o pico de velocidade da estatura, o comprimento da perna e do tronco ocorreram mais tardiamente, cerca de um ano depois, e foram mais lentos quando comparados aos seus pares nãoatletas. Ainda nesse estudo, a idade da menarca e a idade do esqueleto foram consistentes com a maturação somática tardia. ${ }^{7}$ Geithner et al. ${ }^{11}$ observaram que, em meninas americanas e européias, a menarca foi alcançada mais tardiamente em atletas comparativamente às não-atletas, e que houve uma forte associação entre o retardo da menarca e um maior avanço nos níveis competitivos. Esses resultados enfatizam um papel importante dos fatores ambientais, nesse caso o treinamento físico, como um aspecto interventor no processo maturacional. De fato, em resposta ao treinamento físico, parece ocorrer uma diminuição no percentual de gordura corporal acompanhada pelo aumento da massa magra. 12 Particularmente uma diminuição crítica do percentual de gordura corporal parece mediar o aparecimento tardio da menarca. 6

No Brasil, poucos estudos têm descrito aspectos da composição corporal associados à maturação de atletas do sexo feminino. $13 \mathrm{O}$ estudo desses aspectos parece de relevância em atletas de natação que iniciam programas de treinamento físico ainda na infância. Assim, os objetivos do presente estudo foram: descrever os valores médios das variáveis antropométricas peso, estatura e índice de massa corporal, percentual de gordura, massa gorda e massa magra, o somatório e os valores médios das dobras cutâneas tricipital e subescapular; e comparar a idade de menarca de meninas atletas de natação, e de meninas não-atletas.

\section{Métodos}

Foram avaliados 165 indivíduos do sexo feminino (81 não-atletas e 84 atletas de natação), divididos em grupos maturacionais (pré-púberes, púberes e póspúberes) entre os 9 e 17 anos de idade, residentes na cidade do Recife, Pernambuco, Brasil. A formação dos grupos foi aleatória e intencional seguindo os critérios de inclusão como segue: não-atletas = nenhum envolvimento em programa de exercício físico que se caracterize como um treinamento, sendo permitida apenas a participação em aulas de educação física na escola; atletas = estar envolvida em treinamentos há mais de um ano, com uma freqüência mínima de três vezes por semana; desse modo foram convidadas a participar do estudo apenas meninas inscritas em clubes filiados à 
Federação Pernambucana de Natação (FPN).

Um consentimento por escrito, assinado pelo responsável, assim como a própria aceitação das meninas, foram requisitos para participação nesta pesquisa. Este estudo foi aprovado pelo Comitê de Ética em Pesquisa com Seres Humanos da Universidade de Pernambuco (UPE) e seguiu as normas estabelecidas pela Comissão Nacional de Ética e Pesquisa (CONEP), Resolução No. 196/96, sobre pesquisa envolvendo seres humanos.

A estatura e o peso corporal foram determinados através de métodos antropométricos. A estatura foi mensurada entre o plano de referência do solo e o vértex, com o indivíduo descalço, através de um estadiômetro de madeira marca Sunny com precisão de $0,1 \mathrm{~cm}$. O peso corporal foi medido com os indivíduos levemente vestidos (camisa e calção), através de uma balança de marca Filizola com precisão de 100 gramas. O índice de massa corporal (IMC) foi calculado a partir da relação entre o peso, expresso em quilogramas, e o quadrado da altura, expresso em metros. Para obtenção dos valores de dobra cutânea, foi utilizado um plissômetro Lange, de precisão de $0,1 \mathrm{~mm}$, seguindo a técnica sugerida por Lukaski. 14 Foi destacada uma camada de pele e gordura de forma que excluísse o músculo subjacente. O calibrador exercia uma pressão constante de $10 \mathrm{~g} / \mathrm{mm}^{2}$.

Todas as avaliações foram realizadas no hemicorpo direito do avaliado na região tricipital e subescapular e repetida duas vezes em cada local em todos os alunos analisados, ocorrendo uma terceira medição sempre que a diferença entre a primeira e a segunda medição excedia 5\%. No final, era extraída a média aritmética entre os dois valores mais próximos obtidos. Todo o protocolo de medição foi segundo Ross e Jackson. ${ }^{15}$ Para os cálculos da percentagem de gordura, utilizou-se a equação de predição proposta por Slaughter et al.16 Tais equações são ajustadas ao fator maturação, que pode afetar a distribuição de gordura corporal. Assim: $\% \mathrm{GC}=1,33($ tric + sub $)-0,013(\text { Etric }+ \text { sub })^{2}+\mathrm{I}$; sendo I variável com o nível maturacional: -1,7; 3,$4 ;-5,5$ para os estágios pré-púbere, púbere e póspúbere, respectivamente. A massa gorda (MG) foi calculada a partir da relação entre o peso corporal e o percentual de gordura corporal e expressa em kilogramas. A massa corporal magra (MM) foi calculada a partir da diferença entre o peso corporal e a massa gorda e expressa em kilogramas.

Para verificação do estágio maturacional em que cada criança ou adolescente se encontrava foi elaborada uma ficha de inquérito com figuras do desenvolvimento das mamas e da distribuição dos pêlos púbicos, segundo os estágios maturacionais de Tanner. ${ }^{3}$ Os sujeitos deveriam reconhecer a figura que mais se assemelhava ao seu corpo e assinalar em qual dos estágios se encontrava $\left(\mathrm{T}_{1}, \mathrm{~T}_{2}, \mathrm{~T}_{3}, \mathrm{~T}_{4}\right.$ e $\left.\mathrm{T}_{5}\right)$. A auto-avaliação da maturação sexual demons-trou um alto coeficiente de correlação no teste e re-teste ( $r=0,88 ; p<0,05)$. Os estágios de Tanner são os mais utilizados para avaliar a maturação sexual, principalmente em estudos transversais, como acontece com o presente estudo. As meninas que assinalaram os estágios $\mathrm{T}_{1}$ (idade $\left.=9,1 \pm 0,8\right)$ e $\mathrm{T}_{2}$ (idade, $=10,8 \pm 0,9)$ foram consideradas prépúberes. As que assinalaram o estágio $\mathrm{T}_{3}$ (idade $=$ $12,5 \pm 1,0)$ foram consideradas púberes e finalmente os estágio T4 $($ idade $=14,1 \pm 0,7)$ e $\mathrm{T}_{5}($ idade $=15,8$ $\pm 1,0$ ) foram as pós-púberes. A idade de menarca também foi utilizada como indicador de maturação, através do método recordatório segundo Marshall e Tanner, 17 sendo registrada a idade de ocorrência do primeiro fluxo menstrual.

Para a comparação entre os grupos (não-atletas e atletas), em cada fase maturacional, nas variáveis estudadas, utilizou-se o teste "t" de Student para amostras independentes. O nível de significância estabelecido para as análises foi mantido em $5 \%$. Para análise estatística foi utilizado o programa SPSS 10.0 para Windows.

\section{Resultados}

A Tabela 1 apresenta a estatística descritiva e as significâncias entre os valores médios obtidos em cada variável de acordo com a fase maturacional nos grupos de meninas não-atletas e atletas.

No estágio pré-púbere, as meninas atletas não diferiram das não-atletas nas variáveis analisadas $(p>0,05)$ (Tabela 1). Na fase púbere, as atletas, quando comparadas as não-atletas, apresentaram um menor valor médio das dobras cutâneas tricipital e subescapular, assim como no seu somatório ( $\mathrm{SKF}$ ). Ainda na fase púbere, o percentual de gordura corporal (\%GC) e a massa gorda foram menores no grupo de atletas com relação ao grupo não-atleta (Tabela 1).

No estágio pós-púbere, as meninas atletas apresentaram um menor valor médio do peso corporal e da estatura $(p<0,05)$. Da mesma forma, as dobras cutâneas triciptal e subescapular e o SKF foram menores no grupo de atletas. Ainda no estágio póspúbere, os valores médios do \%GC e da massa gorda foram menores nas atletas comparativamente às nãoatletas. Não houve diferença entre os grupos com relação ao Índice de Massa corporal em nenhuma 
Valores médios ( \pm DP) das variáveis antropométricas: peso corporal, estatura, índice de massa corporal (IMC), dobra cutânea (DC) triciptal, dobra cutânea (DC) subescapular, somatório das dobras triciptal e subescapular ( SKF), percentual de gordura (\% GC), massa magra (MM), massa gorda (MG), em meninas atletas e não- atletas, em cada estágio maturacional de Tanner.

\begin{tabular}{|c|c|c|c|c|}
\hline \multirow[t]{2}{*}{ Variáveis } & \multirow[t]{2}{*}{ Grupos } & \multicolumn{3}{|c|}{ Fase maturacional } \\
\hline & & Pré-Púbere & Púbere & Pós-Púbere \\
\hline \multirow[t]{2}{*}{ Peso $(\mathrm{kg})$} & Não-Atletas & $36,89 \pm 8,29(n=13)$ & $53,50 \pm 11,80 \quad(n=44)$ & $60,18 \pm 9,14(n=24)$ \\
\hline & Atletas & $35,81 \pm 5,52(n=25)$ & $50,49 \pm 8,10(n=43)$ & $54,35 \pm 5,85^{*}(n=16)$ \\
\hline \multirow[t]{2}{*}{ Estatura $(\mathrm{cm})$} & Não-Atletas & $141,11 \pm 11,25 \quad(n=13)$ & $158,18 \pm 7,43 \quad(n=44)$ & $161,31 \pm 5,21 \quad(n=24)$ \\
\hline & Atletas & $140,16 \pm 5,78(n=25)$ & $157,20 \pm 7,02(n=43)$ & $156,01 \pm 4,92 *(n=16)$ \\
\hline \multirow[t]{2}{*}{ IMC $\left(\mathrm{kg} / \mathrm{m}^{2}\right)$} & Não-Atletas & $18,37 \pm 2,72(n=13)$ & $21,21 \pm 3,42(n=44)$ & $23,16 \pm 3,64(n=24)$ \\
\hline & Atletas & $18,16 \pm 1,99(n=25)$ & $20,31 \pm 2,10 \quad(n=43)$ & $22,35 \pm 2,47 \quad(n=16)$ \\
\hline \multicolumn{5}{|l|}{ DC } \\
\hline \multirow[t]{2}{*}{ Tríceps (mm) } & Não-Atletas & $16,36 \pm 5,17(n=13)$ & $20,15 \pm 7,06 \quad(n=44)$ & $22,05 \pm 7,87(n=24)$ \\
\hline & Atletas & $14,51 \pm 5,14 \quad(n=25)$ & $15,56 \pm 5,30 *(n=43)$ & $15,09 \pm 4,20 *(n=16)$ \\
\hline \multicolumn{5}{|l|}{ DC } \\
\hline \multirow[t]{2}{*}{ Subescapular (mm) } & Não-Atletas & $14,98 \pm 7,10(n=13)$ & $20,62 \pm 10,35(n=44)$ & $21,42 \pm 10,09(n=24)$ \\
\hline & Atletas & $13,23 \pm 6,30(n=25)$ & $14,76 \pm 5,15^{*}(n=43)$ & $13,84 \pm 2,43 *(n=16)$ \\
\hline \multirow[t]{2}{*}{$\Sigma \mathrm{SKF}(\mathrm{mm})$} & Não-Atletas & $31,34 \pm 11,74(n=13)$ & $40,78 \pm 16,50 \quad(n=44)$ & $43,48 \pm 17,32 \quad(n=24)$ \\
\hline & Atletas & $27,74 \pm 10,78 \quad(n=25)$ & $30,33 \pm 9,84 *(n=43)$ & $28,93 \pm 4,32 *(n=16)$ \\
\hline \multirow[t]{2}{*}{$\% \mathrm{GC}$} & Não-Atletas & $26,00 \pm 6,30(n=13)$ & $28,43 \pm 6,01 \quad(n=44)$ & $28,43 \pm 5,20(n=24)$ \\
\hline & Atletas & $23,80 \pm 6,72(n=25)$ & $25,26 \pm 5,96 * \quad(n=43)$ & $24,72 \pm 2,78^{*} \quad(n=16)$ \\
\hline \multirow[t]{2}{*}{ MM (kg) } & Não-Atletas & $27,04 \pm 5,48 \quad(n=13)$ & $37,94 \pm 7,51 \quad(n=44)$ & $42,82 \pm 5,63(n=24)$ \\
\hline & Atletas & $27,03 \pm 3,08(n=25)$ & $37,48 \pm 5,08(n=43)$ & $40,89 \pm 4,48(n=16)$ \\
\hline \multirow[t]{2}{*}{ MG (kg) } & Não-Atletas & $9,84 \pm 3,76 \quad(n=13)$ & $15,56 \pm 5,56 \quad(n=44)$ & $17,36 \pm 4,94 \quad(n=24)$ \\
\hline & Atletas & $8,78 \pm 3,53(n=25)$ & $13,01 \pm 4,35^{*} \quad(n=43)$ & $13,45 \pm 2,19 *(n=16)$ \\
\hline
\end{tabular}

$* p<0,05$ atletas vs não-atletas; $\mathrm{DC}=$ dobra anterior; SKF $=$; $\mathrm{GC}=$ Gordura Corporal; $\mathrm{MM}=$ Massa Magra;

fase maturacional.

As meninas atletas apresentaram a início da de menarca mais tardiamente que as meninas nãoatletas (atletas $=12,0 \pm 1,04$ e não-atletas $=$ $11,2 \pm 1,37 ; p<0,05)$.

\section{Discussão}

Neste estudo foi feita uma análise descritiva do comportamento das variáveis envolvidas na composição corporal de atletas e não-atletas de natação e comparada a idade da menarca nos diferentes grupos. Para o enquadramento da amostra nos dados normativos, foram utilizados o percentil 75 para o peso corporal e estatura e, para o IMC (valores acima de $24,9 \mathrm{~kg} / \mathrm{m}^{2}$ ), o $\sum \mathrm{SKF}$ (valores acima de $35 \mathrm{~mm}$ ) e o percentual de gordura corporal (até 32\%) sugeridos por Himes e Dietz. 18

Os métodos para avaliação da composição corporal podem ser realizados tanto em laboratório como em campo. 8 Em laboratório, os principais métodos são: densitometria, hidrometria, DEXA e a quantidade total de potássio do corpo. 8 Por outro lado, a utilização de dados antropométricos, como aqueles usados no presente estudo, tem facilitado a avaliação da composição corporal sem a obrigatoriedade de estar em um laboratório.8,9,14 Como exemplo destes métodos podemos citar: dobras cutâneas, percentual de gordura corporal, o índice de 
massa corporal e a bioimpedância elétrica.9,14 Este tipo de avaliação é utilizada normalmente em estudos epidemiológicos ou com grande número de sujeitos participantes. 8,9

No presente estudo, a variação da idade das atletas e não-atletas dentro de cada estágio de maturação foi relativamente ampla. Esse fato é similar com outras observações, sugerindo que a idade cronológica deve ser controlada na comparação dos estágios maturacionais.6,19,20

Entre as atletas do presente estudo, as variáveis peso corporal e estatura apresentaram valores médios inferiores quando comparadas às não-atletas, sendo essas diferenças consideradas significativas apenas na fase pós-púbere. Os resultados desta pesquisa com relação ao peso corporal corroboram estudos previamente realizados, nos quais atletas foram comparadas a não-atletas.21-23 Por outro lado, Benéfice et al.24 observaram que as atletas de natação são freqüentemente mais altas que a população de referência. Os diferentes resultados obtidos podem ser atribuídos às características próprias de cada população estudada, e não ao treinamento físico a que são submetidas, por duas razões: a estatura corporal parece ser mais determinada por fatores genéticos, como a altura dos pais, do que ambientais; 1 e o treinamento de natação parece não promover impacto suficiente para induzir um desgaste articular e comprometer o crescimento ósseo como acontece com outros esportes como a ginástica olímpica e o balé. 25

$\mathrm{Na}$ amostra estudada ambos os grupos apresentaram os valores do IMC, $\sum$ SKF e \%GC dentro dos padrões de normalidade para o estágio maturacional, idade e sexo. Ademais, não houve diferença entre os grupos estudados com relação ao IMC. Nas fases púbere e pós-púbere, o $\sum \mathrm{SKF}$ e o \%GC foram menores nas atletas. Os resultados desse estudo estão de acordo com os relatos de Muller et al.,26 em um estudo envolvendo nível de atividade física e composição corporal de crianças americanas. Malina et al. 2 ressaltam que o treinamento físico é um importante fator de alterações na composição corporal, como dobras cutâneas e percentual de gordura corporal, envolvendo muitas variações entre jovens atletas e não-atletas, o que representaria as diferenças individuais e as alterações adquiridas com a participação esportiva. Várias adaptações fisiológicas ocorrem após um período de treinamento físico. ${ }^{27}$ Uma alteração característica é o aumento da taxa metabólica ou do dispêndio energético após o período de exercício, que vai repercutir diretamente na diminuição do percentual de gordura corporal.28 A mobilização de ácidos graxos dos adipócitos e a utilização desses como fonte de energia no músculo esquelético parecem estar subjacentes às adaptações fisiológicas inerentes ao exercício físico regular. ${ }^{28}$

A gordura corporal localizada subcutaneamente é o maior depósito de gordura corporal, podendo representar cerca de $60 \%$ da quantidade de gordura total do corpo. ${ }^{9}$ Os locais mais utilizados para avaliação da quantidade de gordura subcutânea são as regiões tricipital e subescapular. $9,17,26$ As dobras cutâneas subescapular e tricipital também podem ser utilizadas para distinguir entre gordura central e gordura periférica. ${ }^{2}$ No presente estudo, as atletas apresentaram um menor valor médio de dobras cutâneas tricipital e subescapular comparativamente às não-atletas. É interessante observar que os valores médios em cada fase maturacional praticamente não se alteram, sugerindo, dessa forma, uma estabilidade na deposição de gordura subcutânea periférica e central em meninas atletas.

Com relação à massa corporal magra, não foram evidenciadas diferenças entre atletas e não-atletas em nenhuma das fases maturacionais, o que pode estar associado ao tipo de modalidade praticada. A natação parece não favorecer um ganho acentuado de massa muscular, pois isso implicaria numa menor flutuabilidade e prejudicaria o desempenho.24 Avlonitou et al.,8 estudando os efeitos da prática competitiva da natação sobre a composição corporal, observaram que a densidade óssea e a massa corporal magra nos membros inferiores não foram afetadas pelo treinamento da natação, porém ocorreu uma diminuição na gordura corporal.

No presente estudo, o grupo de atletas apresentou valores médios menores com relação à quantidade de massa gorda comparativamente aos seus pares não-atletas. Essa diferença foi significativa nas fases púbere e pós-púbere, o que pode estar relacionado com o início da puberdade e com o percentual de gordura aumentado nessas fases. Apesar da diferença entre as atletas e as não-atletas, ambos se apresentam dentro dos valores de normalidade quando os resultados são comparados com dados normativos. 18

O início da menarca das atletas ocorreu mais tardiamente comparativamente às não-atletas. Esses resultados vão de encontro aos de Geithner et al. ${ }^{11}$ com atletas de remo e natação. Os autores não encontraram diferença entre a idade de ocorrência da menarca entre o grupo de atletas e seus pares nãoatletas. Malina et al. ${ }^{2}$ verificaram que atletas de natação e de remo apresentaram início da menarca pelo menos um ano depois $(13,6 \pm 0,12$ anos) das não-atletas $(12,5 \pm 1,0$ anos $)$. Hitchen et al.29 observaram que em meninas americanas e européias, a 
menarca foi alcançada mais tardiamente em atletas comparativamente às não-atletas, assim como uma forte associação entre o retardo da menarca e um maior avanço no rendimento competitivo. Parece que a quantidade de gordura corporal e a sobrecarga fisiológica de programas de treinamento podem influenciar o perfil hormonal e, como conseqüência, ocorrer um atraso no aparecimento da menstruação.1,6 De fato a composição corporal pode sofrer influência de fatores ambientais, como dieta e exercício físico, genéticos e hormonais. De uma perspectiva endócrina, os níveis de secreção de hormônios esteróides, responsáveis pelas alterações somáticas e fisiológicas no processo maturacional, podem também sofrer a influência de fatores como intensidade de esforço físico e aporte adequado de nutrientes. 1,6

Assim, no presente estudo foram avaliados de forma descritiva alguns componentes da composição corporal de meninas atletas de natação e comparados com seus pares não-atletas. Observou-se que as alterações no percentual de gordura corporal, nos valores médios de dobras cutâneas e na quantidade de massa gorda foram menores nas atletas. Esses resultados podem ser associados à prática regular de exercícios físicos com fins competitivos, onde a intensidade do esforço induz alterações fisiológicas importantes.

Também foi observado que a idade da menarca ocorreu mais tarde nas atletas. É possível que haja uma correlação entre alguns componentes da composição corporal e o processo maturacional. O fato de este estudo ter sido de natureza transversal pode ter comprometido a consistência das comparações. Um estudo longitudinal seria o ideal, quando se leva em consideração as mudanças que ocorrem durante o crescimento.

\section{Referências}

1. Rogol AD, Roemmich JN, Clark PA. Growth at puberty. J Adolesc Health. 2002; 31 (6 Suppl): 192-200.

2. Malina RM, Koziel S, Bielicki T. Variation in subcutaneous adipose tissue distribution associated with age, sex, and maturation. Am J Hum Biol. 1999;11:189-200.

3. Tanner JM. Growth and adolescence. 2nd ed. Oxford: Blakwell; 1962.

4. Spear BA. Adolescent growth and development. J Am Diet Assoc. 2002; 102 (Suppl): S23-9.

5. Engelbregt MJ, van Weissenbruch MM, Lips P, van Lingen A, Roos JC, Dalemarre-van de Waal HA. Body composition and bone measurements in intra-uterine growth retarded and early postnatally undernourished male and female rats at the age of 6 months: comparison with puberty. Bone. 2004; 34: 180-6.

6. Claessens AL, Bourgois J, Beunen G, Philippaerts R, Thomis M, Lefreve J, Loos RJ, Vrijens J. Age at menarche in relation to anthropometric characteristics, competition level and boat category in elite junior rowers. Ann Hum Biol. 2003; 30: 148-59.

7. Thomis M, Claessens AL, Lefevre J, Philippaerts R, Beunen GP, Malina RM. Adolescent growth spurts in female gymnasts. J Pediatr. 2005; 146: 239-44.

8. Avlonitou E, Georgiou E, Douskas G, Louizi A. Estimation of body composition in competitive swimmers by means of three different techniques. Int J Sports Med. 1997; 18: 3638 .

9. Lohman TG. Exercise training and body composition in childhood. Can J Sport Sci. 1992; 17: 284-7.

10. Theintz GE, Howald GE, Weiss U, Sizonenko PC. Evidence for a reduction of growth potential in adolescent female gymnasts. J Pediatr. 1993; 122: 306-13.
11. Geithner CA, Woynarowska B, Malina RM. The adolescent spurt and sexual maturation in girls active and not active in sport. Ann Hum Biol. 1998; 25: 415-23.

12. Leandro C, Guerra S, Duarte JA, Mota J. Maturação, composição corporal e aptidão cardiorespiratória em crianças e adolescentes na área do Grande Porto, Portugal. Rev Bras Saúde Matern Infant. 2001; 5: 249-56.

13. Biassio LG, Matsudo SMM, Matsudo VHR. Impacto da menarca nas variáveis antropométricas e neuromotoras da aptidão física, analisado longitudinalmente. Rev Bras Ci Mov. 2004; 12: 97-101.

14. Lukaski HC. Methods for the assessment of uman Body Composition: traditional and new. Am J Clin Nutr. 1987; 46: 537-56.

15. Ross RM, Jackson AS. Exercise concepts, calculations, and computer applications. Carmel, Indiana: Benchmark; 1990.

16. Slaughter MH, Lohman TG, Boileau RA, Horswill CA, Stillman RJ, van Loan MD, Bemben DA. Skinfold equations for estimation of body fatness in children and youth. Hum Biol. 1988; 60: 709-23.

17. Marshall WA, Tanner JM. Puberty. In: Falkner F, Tanner JM, editors. Human growth. New York: Plenun Press; 1986. v. 2, p.171-210.

18. Himes JH, Dietz WH. Guidelines for overweight in adolescent preventive services: recommendations from an Expert Committee. Am J Clinical Nutr. 1994; 59: 307-16.

19. Mota J, Guerra S, Leandro C, Pinto A, Ribeiro JC, Duarte JA. Association of maturation, sex, and body fat in cardiorespiratory fitness. Am J Hum Biol. 2002; 14: 70712. 
20. Proctor DN, Melton LJ, Khosla S, Crowson CS, O'Connor MK, Riggs BL. Relative influence of physical activity, muscle mass, and strength on bone density. Osteoporos Int. 2000;11: 944-52.

21. Andersen LK, Ghuesquiere J. Sex differences in maximal oxygen uptake, heart rate, and oxygen pulse at 10 and 14 years in Nowegian children. In: Reybrouck T, Ostyn M, editors. The KU-Leuven contributions to pediatric work physiology. Leuven; 1989.

22. Claessens AL, Lefreve J, Beunen G, Malina RM. The contribution of anthropometric characteristics to performance scores in elite female gymnasts. J Sports Med Phys Fitness. 1999; 39: 355-60.

23. Engelhardt L, Willers BC, Pelz L. Sexual maturation in East German girls. Acta Paediatr. 1995; 84: 1362-5.

24. Benéfice E, Mercier J, Guerin MJ, Préfault $\mathrm{MH}$ Differences in aerobic and antropometric characteristics between peripubertal swimmer and no swimmers. Int J Sports Med. 1990; 11: 456-60.

25. Beunen G, Malina RM, Lefevre J, Claessens AL, Renson R, Simons J, Maes H, Vansreusel B, Lysens R. Size, fatness and relative fat distribution of males of contrasting maturity status during adolescence and adults. Int J Obes. 1994; 18: 670-8.

Recebido em 27 de agosto de 2006

Versão final apresentada em 9 de janeiro de 2007

Aprovado em 10 de fevereiro de 2007
26. Muller WH, Harrist RB, Bruce A, Doyle RB, Ayrs CL, Labarthe DR. Body measurement variability, fatness, and fat-free mass in children 8, 11 and 14 years of age: project heart beat. Am J Hum Biol. 1999; 14: 69-78.

27. Bass S, Bradney M, Pearce G, Hendrich E, Inge K, Stuckey S, Lo SK, Seeman E. Short stature and delayed puberty in gymnasts: influence of selection bias on leg length and the duration of training on trunk length. J Pediatr. 2000; 136: 149-55.

28. Papanek PE. The female athlete triad: an emerging role for physical therapy. J Orthop Sports Phys Ther. 2003; 33: 594614.

29. Hitchen PJ, Jones MA, Stratton G. Maturity and gender effects on selected physical fitness parameters in high school age children. J Sport Sci. 1999; 17: 18-9. 